\title{
QUALIDADE DE VIDA DO PACIENTE SUBMETIDO À MICROCIRURGIA ENDOSCÓPICA TRANSANAL (TEM)
}

\author{
Quality of life after Transanal Endoscopic Microsurgery (TEM)
}

\author{
Roberto da Silveira MORAES ${ }^{1}$, Marcos Vinícius ZANCHET $^{1}$, Wagner Herbert SOBOTTKA ${ }^{1}$, \\ João Henrique Felício de LIMA ${ }^{1}$, Glauco Afonso MORGENSTERN ${ }^{1}$, Osvaldo MALAFAIA ${ }^{1}$, \\ Gerhard BUESS ${ }^{2}$, Júlio Cezar Uili COELHO ${ }^{1}$
}

ABCDDV/522

Moraes RS, Zanchet MV, Sobottka WH, Lima JHF, Morgenstern GA, Malafaia O, Buess G, Coelho JCU. Qualidade de vida do paciente submetido à Microcirurgia Endoscópica Transanal (TEM). ABCD Arq Bras Cir Dig 2007;20(1):34-7.

RESUMO - Racional - A Microcirurgia Endoscópica Transanal (TEM) é procedimento minimamente invasivo para o tratamento de tumores retais selecionados. Atualmente, existe crescente interesse médico na medida quantitativa da qualidade de vida. Objetivo - Avaliar a qualidade de vida dos pacientes submetidos a TEM no Serviço de Cirurgia do Aparelho Digestivo no Hospital de Clínicas da Universidade Federal do Paraná. Métodos - Trata-se de um estudo observacional prospectivo e de coorte da avaliação da qualidade de vida após TEM. Trinta e quatro pacientes responderam a um questionário composto de 14 questões, abordando aspectos pós-operatórios e laborais. Dirigiam-se elas para levantar dados principalmente sobre: o consentimento informado; a dor experimentada após a operação; a capacidade de o paciente caminhar no período pós-operatório; o período para retorno às atividades habituais; a satisfação com a ausência de cicatriz pós-operatória; a incontinência no pós-operatório; se recomendaria a operação a um familiar ou conhecido. Resultados - Todos os 34 pacientes relataram ter sido adequadamente informados sobre o procedimento. Ausência de dor pós-operatória foi observada em $82,5 \%$ e todos se mostraram capazes de deambular no $1^{\circ}$ dia do pós-operatório. O retorno às atividades habituais deu-se em média sete dias após o procedimento. Somente cinco pacientes $(14,70 \%)$ apresentaram incontinência fecal transitória, não maior que uma semana. Três pacientes $(8,82 \%)$ necessitaram de re-internação, sendo dois por tumores residuais e outro por recidiva tumoral. Dois pacientes (5,88\%) referiram modificação temporária na vida sexual após a cirurgia e 97,05\% indicariam a TEM a um familiar ou amigo. O período médio de internação foi de três dias. Conclusão - Os pacientes apresentaram boa evolução, com pouca dor pós-operatória, curto período de internação e baixo índice de complicações, mostrando satisfação e adequada qualidade de vida com a TEM.

DESCRITORES - Microcirurgia Endoscópica Transanal (TEM). Qualidade de vida. Pós-operatório. Tumores retais.

\section{INTRODUÇÃO}

A Microcirurgia Endoscópica Transanal (TEM) foi desenvolvida em 1983 por Gerhard Buess ${ }^{13}$ e corresponde a um dos mais recentes avanços no campo da cirurgia minimamente invasiva para o tratamento dos tumores do reto. A TEM proporciona o tratamento de lesões retais selecionadas com baixa morbi-mortalidade e resultados semelhantes ao da cirurgia radical convencional.

Atualmente, existe a tendência em se avaliar a qualidade de vida pós-operatória nas diversos procedimentos cirúrgicos. Não há qualquer trabalho, conforme as fontes de dados do MEDLINE e da literatura médica brasileira, que avalie especificamente e de maneira objetiva a qualidade de vida após a TEM. Igualmente, a maioria dos trabalhos com essa finalidade são adaptações de publicações estrangeiras, nem sempre aplicáveis de modo adequado em nosso meio e para todos os procedimentos cirúrgicos.

O objetivo do presente estudo foi o de realizar análise objetiva, qualitativa e quantitativa dos aspectos clínicos

Trabalho realizado no Serviço de Cirurgia do Aparelho Digestivo do Hospital de Clínicas da Universidade Federal do Paraná (UFPR) ${ }^{1}$ e Department of General Surgery, Eberhard-Karls University, Tuebingen, Germany ${ }^{2}$.

Endereço para correspondência: Dr. Roberto da Silveira Moraes, e-mail: rdsmoraes@ig.com.br e laborais de pacientes submetidos à TEM e operados no Serviço de Cirurgia do Aparelho Digestivo do Hospital de Clínicas da Universidade Federal do Paraná, Curitiba, PR. Esses dados refletem a qualidade de vida no pós-operatório e permitem inferir a aceitabilidade do procedimento como nova modalidade cirúrgica para tratamento de tumores retais selecionados.

\section{MÉTODOS}

Trata-se de um estudo observacional prospectivo e de coorte da avaliação da qualidade de vida após a TEM. Todos os dados foram obtidos prospectivamente pelos pesquisadores na avaliação dos pacientes por meio da aplicação de um questionário, dados estes registrados em planilha eletrônica (Microsoft Excel ${ }^{\circledR}$ ), conferidos e transportados para o software Statistica ${ }^{\circledR}$.

Ao todo, 34 pacientes, 17 mulheres e 17 homens, com idade entre 24 e 89 anos, responderam ao questionário composto por 14 itens, abordando aspectos pós-operatórios e laborais (Figura 1) sobre: o consentimento informado; a dor experimentada após a operação; o período de internação; a capacidade de o paciente caminhar no período pós-operatório; o período para retorno às atividades habituais; a satisfação 
com a ausência de cicatriz pós-operatória; a incontinência no pós-operatório; se recomendaria a operação a um familiar ou conhecido; e aspectos negativos do procedimento.

A diferença entre as variáveis contínuas foi avaliada pelo teste de Mann-Whitney, considerando a natureza assimétrica de sua distribuição. A diferença entre as freqüências obtidas nas respostas elaboradas foi avaliada por meio do teste para diferença entre proporções.

O tamanho da amostra foi estimado considerando-se erro do tipo I de $5 \%$ (alfa) e do tipo II de $10 \%$, com poder de teste estimado mínimo de $90 \%$.

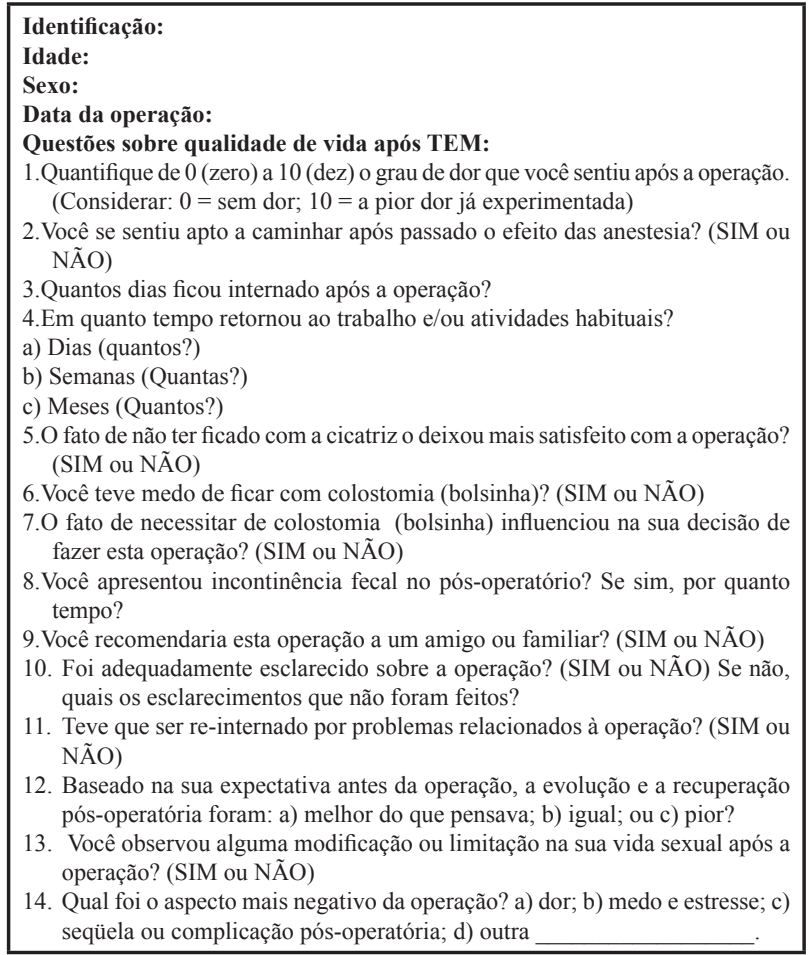

FIGURA 1 - Questionário sobre qualidade de vida após a TEM

\section{RESULTADOS}

Os pacientes incluídos no estudo apresentaram média global de idade de 60 anos, sendo de 62,7 para os homens e 57,4 para as mulheres.

Com relação à dor experimentada após a operação, 28 pacientes $(82,35 \%)$ referiram ausência de dor no período pós-operatório; dois $(5,88 \%)$ referiram dor que foi por eles classificada como grau 3; mais dois $(5,88 \%)$ como grau 5 ; um $(2,94 \%)$ como grau 6 e mais um $(2,94 \%)$ como grau 9 $(P<0,01)$ (Gráfico 1).

A capacidade de o paciente caminhar no período pósoperatório após o término do efeito da anestesia foi verificada nos 34 pacientes interrogados (100\%) e foi normal.

O período de internação foi em mediana de 3 dias, variando de 1 a 8 dias. Observa-se maior tempo de internação entre os pacientes que apresentaram dor no período pósoperatório, e essa relação apresentou significância estatística $(P=0,006)$ (Gráfico 2).

$\mathrm{O}$ período para o retorno às atividades habituais foi em

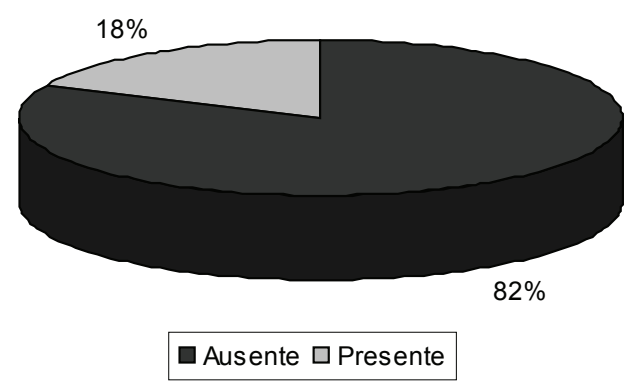

GRÁFICO 1 - Dor pós-operatória

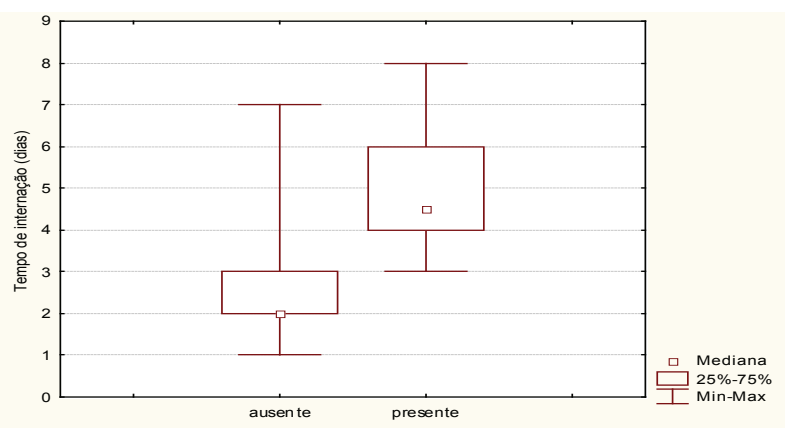

GRÁFICO 2 - Tempo de internação de acordo com a presença ou não de dor no período pós-operatório

média de sete dias, variando de dois a 20 dias. Observou-se diferença estatisticamente significativa no que se refere a este tempo entre os pacientes que apresentaram ou não dor no período pós-operatório, que foi em média de sete dias (dois a 15 dias) para aqueles pacientes sem dor; e de 12,5 dias (sete a 20 dias) para os pacientes com dor $(P=0,005)$.

Todos os pacientes referiram satisfação com a ausência de cicatriz pós-operatória, e 29 pacientes $(85,29 \%)$ referiram sentir medo de necessitar de colostomia no pós-operatório. Vinte e sete pacientes $(79,41 \%)$ referiram que o fato de não necessitar de colostomia influenciou positivamente na decisão pelo procedimento, contra sete pacientes $(20,58 \%)$ que se mostraram indiferentes a este aspecto $(P<0,01)$.

Somente cinco pacientes $(14,70 \%)$ apresentaram algum tipo de incontinência no pós-operatório (Gráfico 3), na maioria dos casos temporária e inferior a um mês após a operação.

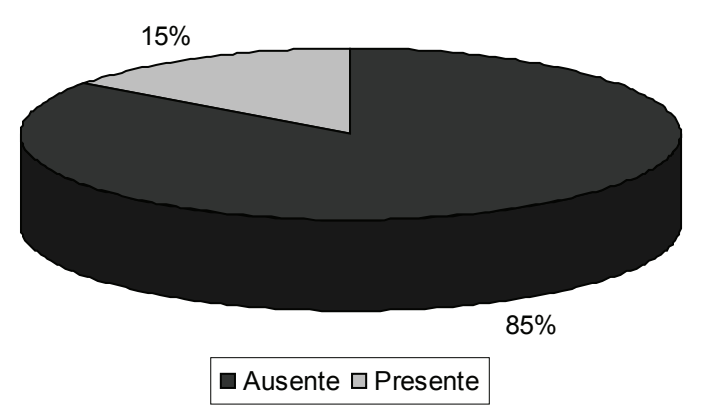

GRÁFICO 3 - Freqüência de incontinência fecal no pósoperatório 
A quase totalidade dos pacientes (97,05\%) recomendaria a TEM a um familiar ou conhecido e todos foram adequadamente esclarecidos sobre ela no pré-operatório, sendo que $97,05 \%$ deles referiram evolução pós-operatória superior ao esperado. Somente dois pacientes $(5,88 \%)$ referiram modificação ou limitação temporária na vida sexual após a operação $(P<0,01)$.

Apenas três pacientes $(8,82 \%)$ necessitaram de re-internação por problemas relacionados à TEM, sendo dois por tumores residuais e outro por recidiva tumoral.

Com relação aos aspectos negativos do procedimento, 11 pacientes $(32,35 \%)$ não apontaram nenhum, quatro $(11,76 \%)$ referiram a dor como o aspecto mais negativo, nove $(26,47 \%)$ medo, e dez $(29,41 \%)$ outros aspectos como os exames pré-operatórios, o preparo intestinal, vômitos após a anestesia, sangramento, incontinência para flatos e a própria anestesia.

\section{DISCUSSÃO}

Existe hoje crescente interesse de médicos e pesquisadores em avaliar o conceito de qualidade de vida e transformálo em medida quantitativa que possa ser usada em ensaios clínicos e modelos econômicos para fins comparativos ${ }^{1}$. Por ser a TEM um método novo na prática proctológica, ela carece de estudos com essa finalidade.

Além disso, outras dificuldades surgem quando se pretende maior aprofundamento nessa questão. Os estudos que propõem analisar a qualidade de vida são em geral direcionados à doenças crônicas, como a artrite reumatóide, e não permitem aplicação apropriada a pacientes de status cirúrgico pós-operatório ${ }^{2,3,4,5,6,7,8}$. Ademais, boa parte deles são reproduções ou adaptações de questionários estrangeiros, nem sempre adequados à realidade cultural brasileira.

Buscou-se elaborar um questionário que fosse simplificado, compreensível e reprodutível, e cuja aplicabilidade não se limitasse a avaliar o paciente no pós-operatório, mas também que permitisse verificar a aceitação da TEM e alguns de seus resultados a curto e médio prazo. Dentre esses resultados, vem ganhando destaque a avaliação da função esfincteriana após a TEM ${ }^{9,10,11,12,14}$.

Foi verificada alteração da função esfincteriana clinicamente significativa em $14,7 \%$ dos pacientes após a operação. Outros autores relatam índices que variam de 7,5\%9 a $37 \%{ }^{11}$. Dos cinco pacientes que apresentaram algum tipo de incontinência, três tiveram incontinência fecal transitória. Destes, dois tiveram melhora espontânea após uma semana e um após 20 dias de fisioterapia esfincteriana. Um paciente persiste com incontinência a flatus, mas já apresentava esta disfunção previamente à operação.

Estudos prospectivos utilizando manometria e ultrassonografia endoanal pré e pós-operatórias mostram que a dilatação anal durante o procedimento pode ocasionar neuropraxia do esfíncter anal interno, podendo levar a incontinência temporária ${ }^{9}$.Contudo, de modo global, a TEM parece afetar pouco a continência dos pacientes ${ }^{11}$.

Com relação à dor pós-operatória, $17,64 \%$ dos pacientes apresentaram algum tipo de dor, classificada como leve $(5,88 \%)$, moderada $(8,82 \%)$ ou intensa $(2,94 \%)$ naqueles submetidos a anastomoses retoanais. Todos sentiram-se aptos a deambular após o término do efeito da anestesia. Verificou-se relação estatisticamente significativa entre a presença de dor pós-operatória e um maior tempo de internação. Igualmente, foi observado maior tempo para o retorno ao trabalho e às atividades habituais para os pacientes com dor no período pós-operatório.

Os principais aspectos positivos da operação foram: a ausência de cicatriz pós-operatória e o fato de não necessitarem de colostomia. Todos informaram que foram adequadamente esclarecidos sobre a TEM no pré-operatório, e referiram evolução pós-operatória superior ao esperado.

\section{CONCLUSÃO}

Os pacientes apresentaram boa evolução após a TEM e a ausência de dor pós-operatória parece ter sido o indicador mais fortemente associado com estes aspectos. A quase totalidade dos pacientes mostrou-se satisfeita com o procedimento. Com a TEM, os pacientes mantiveram preservada sua qualidade de vida.

Moraes RS, Zanchet MV, Sobottka WH, Lima JHF, Morgenstern GA, Malafaia O, Buess G, Coelho JCU. Quality of life after transanal endoscopic microsurgery (TEM). ABCD Arq Bras Cir Dig 2007; 20(1):34-7.

ABSTRACT - Background - Transanal Endoscopic Microsurgery (TEM) is a minimal invasive procedure for the treatment of rectal tumors. Nowadays there has been an increasing medical interest regarding quantitative measurements pertaining quality of life. Aim - To evaluate life quality of patients submitted to TEM at Hospital de Clínicas of the Federal University of Paraná in the Department of Surgery of the Digestive Tract. Methods - A prospective observational cohort study was done to evaluate the quality of life after TEM. Thirty-four patients answered a questionnaire composed of 14 questions concerning postoperative and labor issues. Questions concerned primarily about: informed consent; pain felt after surgery; patients capacity to walk during the postoperative state; period of return to normal activities; satisfaction regarding the absence of postoperative scars; postoperative incontinence, as well as the recommendation of this surgery to family members or friends. Results - All thirty-four patients claimed to be adequately informed about the procedure. The absence of postoperative pain was observed in $82.5 \%$, and all demonstrated the capacity to walk in the first postoperative day. In average, returning to normal activities began after the seventh day of surgery. Only five patients (14.70\%) experimented transitory fecal incontinence, enduring no longer than a week. Three patients $(8.82 \%)$ were later readmitted to the hospital: two with residual tumors and the other for tumor recurrence. Two patients $(5.88 \%)$ complained about temporary modification in his/her sexual life after the surgery, and $97.05 \%$ would indicate TEM for his/her family members or friends. The average time period of admission was of three days. Conclusion - Patients presented a good evolution, with little postoperative pain, and a low complication index, demonstrating satisfactory and adequate quality of life after TEM.

HEADINGS - Microsurgery endoscopy gastrointestinal. Quality of life. Postoperative pain. Rectal neoplasms. 


\section{REFERÊNCIAS}

1. Ciconelli RM, Bosi Ferraz M, Santos W, Meinão I, Quaresma MR. Tradução para a língua portuguesa e validação do questionário genérico de avaliaçãode qualidade de vida SF-36 (Brasil SF-36). Rev Bras Reumatol 39 (3): 143-50, 1999.

2. Bowling A, Brazier J. Quality of life in social science and medicine. Soc Sci Med 41: 1337-8, 1995 .

3. Guillemin F, Bombardier C, Beaton D: Cross-cultural adaptation of healthrelated qualitiy of life measures: literature review and proposed guidelines. J Clin Epidemiol 46: 1417-1432, 1993.

4. Gullemin F: Cross-cultural adaptation and validation of health status measures. Scan J Rheumatol 24:61-3, 1995.

5. Ware JE, Sherbourne CD. The MOS 36 Item Short-Form Health Survey (SF-36). 1. Conceptual framework and item selection. Med Care 30: 473-83, 1992.

6. Ware JE, Gandek B, IQOLA Project Group. The SF-36 health survey: development and use in mental health research and the IQOLA project. Int J Med Health 23: 49-73, 1994.

7. Ware JE, Kosinski M, Keller ED. The SF-36 Physical and Mental Summary Scales: A user's Manual, Boston, MA. The Health Institute, 1994.

8. Meenan RF, Mason JH, Anderson JJ et al. The content and properties of revised and expanded Arthritis Impact Measurement Scales Health Status Questionnaire. Arthirtis Rheum 35: 1-10, 1992
9. Gracia Solanas JA, Ramírez Rodríguez JM, Aguilella Diago V, Elía Guedea M, Martínez Díez M. A prospective study about functional and anatomic consequences of transanal endoscopic microsurgery. Rev Esp Enferm Dig, 98 (4): 234-40, 2006.

10. Cataldo PA, O'Brien S, Osler T. Transanal endoscopic microsurgery: a prospective evaluation of functional results. Dis Colon Rectum 48 (7): 1366-71, 2005.

11. Dafnis G, Påhlman L, Raab Y, Gustafsson UM, Graf W. Transanal endoscopic microsurgery: clinical and functional results. Colorectal Dis, 6 (5): 336-42, 2004.

12. Coelho, JCU, Parolin MB, Baretta GAP, Pimentel SK, De Freitas, AC T, Colman D. Qualidade de vida do doador após transplante hepático intervivos. Arq Gastroenterol 42 (2): 83-8, 2005.

13. Moraes RS, Campos ACL, Telles JEQ, Coelho JCU. Transanal endoscopic microsurgery (TEM): a new concept of minimally invasive treatment of rectal tumors. ABCD Arq Bras Cir Dig 2003; 16(1):3-5.

14. Moraes RS, Buess G, Campos, Malafaia O, Marinho Jr CH, Coelho JCU. Transanal endoscopic microsurgery in the treatment of superficial rectal câncer. Report of a case. ABCD Arq Bras Cir Dig 2002; 15:36-9.

Conflito de interesse: não há Fonte financiadora: não há Recebido para publicação em: 02/07/2006 Aceito para publicação em: 19/10/2006 